\title{
Falar e Mostrar para Provar: Uma Contribuição Teórica sobre a Utilização dos Gestos Ostensivos Wittgensteinianos como Auxiliares na Prova Matemática
}

(Speak and Show to Prove: A Theoretical Contribution on the Use of Ostensible Gestures Wittgensteinians as Auxiliaries in Mathematical Proof)

\section{MARCELO DE SOUSA OLIVEIRA ${ }^{1}$ e MARISA ROSÂNI ABREU DA SILVEIRA $^{2}$}

\footnotetext{
${ }^{1}$ Universidade Federal do Sul e Sudeste do Pará (moliveira@ unifesspa.edu.br)

${ }^{2}$ Universidade Federal do Pará (marisabreu@ufpa.br)
}

\begin{abstract}
Resumo. Este artigo, de natureza teórica, tem o objetivo de tratar da utilização de gestos ostensivos como auxiliares do professor no processo de mostrar provas matemáticas aos seus alunos. Para tanto, discorremos sobre alguns conceitos utilizados nas aulas de matemática, tais como o conceito de prova e de demonstração que são passíveis do uso de gestos ostensivos na busca do sentido de proposições matemáticas. Nossas análises estão apoiadas na filosofia de Ludwig Wittgenstein, em alguns textos de comentadores de sua filosofia e de alguns educadores matemáticos que a usam como fundamentação filosófica.
\end{abstract}

Abstract. This article, fron theoretical nature, which goal is to discuss the usage of ostensible gestures as teacher's assistants in the process of exposing mathematical proofs to students. Therefore, we discuss some concepts used in math's classes, such as theories and demonstrations' proof, which are susceptible of using ostentatious gestures searching the meaning of mathematical propositions. Our analysis is based on the Ludwig Wittgenstein's philosophy, as well as on some philosophy commentators's of his texts and some mathematic educators who employ it as a philosophical foundation.

Palavras-chave: prova, demonstração, ensino ostensivo, definição ostensiva

Keyword: proof, demonstration, ostensible education, ostensible definition

\section{Introdução}

Afirmações que destacam a importância do desenvolvimento da habilidade de leitura, de interpretação e de utilização da linguagem matemática por parte dos alunos, tais como lidar com equações, gráficos, diagramas, fórmulas, tabelas, etc., estão presentes em documentos oficiais de orientação curricular (BRASIL, 2000).

A linguagem matemática tem sido focalizada como objeto de pesquisa no campo da educação matemática e essas pesquisas têm contribuído para compreendermos como os processos linguísticos interferem no ensino e na aprendizagem da matemática.

A filosofia de Wittgenstein tem sido utilizada como embasamento filosófico para responder questões desse tipo em algumas linhas de pesquisa, tais como a filosofia da educação matemática, a modelagem matemática e a linha estudos da linguagem matemática.

Vilela (2009) se utilizou da filosofia de Wittgenstein para compreender os usos que tem sido feito da expressão matemática e o que esses usos indicam no âmbito da educação matemática. 
Na linha de pesquisa da modelagem, Oliveira (2010) analisou a produção de sentidos pelos alunos no desenvolvimento de atividades de modelagem; a pesquisa de Tortola (2012) investigou os usos que estudantes de anos iniciais do Ensino Fundamental fazem da linguagem para o desenvolvimento de modelos matemáticos; na mesma direção, Merli (2012) realizou um estudo sobre o uso da linguagem na construção de modelos matemáticos clássicos e modelos fuzzy; já Souza (2013), a partir da análise de um levantamento bibliográfico sobre os esquemas do processo de modelagem, utilizou o conceito wittgensteiniano de semelhança de família para identificar convergências entre os esquemas e propôs esquemas-síntese.

Embora as pesquisas citadas acima tenham utilizado conceitos da filosofia de Wittgenstein, a preocupação com questões relativas à linguagem matemática é secundária, como as da linha da modelagem ou inexistente, como a pesquisa de Vilela (2009) que utilizou os conceitos de uso e de semelhança de família para empreender a análise sobre as adjetivações do termo matemática em trabalhos relativos à Etnomatemática.

Dentre as pesquisas que tem a preocupação primordial com questões relativas ao uso da linguagem matemática em situações de ensino e de aprendizagem, destacamos a pesquisa de Silva (2011) que investigou o aprendizado de regras matemáticas por crianças da $4^{\mathrm{a}}$ série no estudo da divisão; o estudo de Lacerda (2010), que analisou o processo de interpretação e de comunicação a partir dos dizeres e das produções escritas dos alunos na resolução de problemas, empreendidas individualmente e em duplas.

Nessa direção, o presente estudo visa contribuir com as pesquisas já realizadas sobre o tema no sentido de oferecer contribuições teóricas para uma compreensão de questões relativas ao uso da linguagem no processo de prova de proposições matemáticas no âmbito escolar.

Explicitaremos de maneira geral o significado de palavras relacionadas à argumentação matemática, já que tanto no campo científico da matemática quanto no campo educacional, onde atua o professor de matemática, é comum tratarmos de termos como axiomas, teoremas, proposições, prova, demonstração, generalização, dentre outros, com suas especificidades em relação ao sentido que é atribuído em cada um dos campos.

Algumas pesquisas em educação matemática indicam que muitos professores da educação básica possuem conhecimento superficial em relação a esses conceitos e até 
mesmo os confundem em alguns casos, como a distinção entre uma demonstração, uma generalização e uma argumentação (SALES; PAIS, 2009; ALMOULOUD; FUSCO, 2010; ALMOULOUD et al., 2012). Já o pesquisador matemático possui mais clareza, uma vez que estes conceitos são parte essencial da sua prática profissional.

Neste texto procuramos discorrer sobre a especificidade do ato de provar a veracidade ou validade de proposições matemáticas por meio de explicações e argumentações em que os gestos ostensivos do professor são usados para facilitar a compreensão da prova pelo aluno. Nesse sentido, temos o objetivo de refletir sobre o uso dos gestos ostensivos na efetivação da prova no ensino da matemática amparados na filosofia de Wittgenstein.

Para tanto, iniciaremos expondo alguns pontos de vista sobre a argumentação matemática e em seguida discutiremos algumas aplicações dos gestos ostensivos wittgensteinianos no ensino e na aprendizagem da matemática; posteriormente, a partir da exposição de exemplos, utilizaremos essas ideias para empreender nossa análise a respeito da utilização dos gestos ostensivos no processo de prova de proposições matemática e as implicações para o tema aqui delimitado.

\section{Alguns pontos de vista sobre a argumentação matemática}

Explicação, argumentação, prova e demonstração são palavras muito presentes na aula de matemática e que possuem nesse ambiente sentido bem específico, em função da prática profissional do professor, sobretudo por implicação de questões relacionadas à natureza do conhecimento matemático. Neste sentido, para compreendermos o uso destas palavras pelo professor buscamos elucidar tais conceitos baseados em um referencial teórico que julgamos ser pertinente, a saber, o pensamento do filósofo Ludwig Wittgenstein e de alguns educadores matemáticos.

Segundo Almouloud et al. (2011) com base em Balacheff (1982), quando o professor tem a finalidade de comunicar o caráter de verdade de um enunciado matemático aos alunos, utiliza uma explicação reconhecida como convincente pela comunidade que se constitui como prova para esta comunidade. Balacheff (1987) nos dá elementos para distinguirmos diferentes níveis do processo argumentativo no ensino da matemática, tais como explicação, prova ou demonstração. A explicação está intimamente ligada ao sujeito locutor e tem por finalidade comunicar o caráter de verdade de um enunciado matemático; as provas são explicações aceitas por um grupo 
social em momento específico. Se a prova se refere a enunciados matemáticos, então se trata de uma demonstração.

As demonstrações são provas particulares com as seguintes características: são reconhecidas pelo matemático profissional; respeitam a regra de partir de axiomas enunciados provenientes da experiência tidos como verdadeiros, mediante a intuição e a evidência - e deduzir outros enunciados ou partir dos demonstrados anteriormente, obedecendo a regras lógicas para chegar aos teoremas ${ }^{1}$; trabalham dentro de um estatuto teórico não pertencente ao mundo sensível. A demonstração tem por finalidade uma única verdade que não deixa margem para dúvida a respeito de sua validação.

Neste caso pode-se afirmar que a demonstração é teórica e tem caráter científico. Já a prova e a argumentação não necessitam de muito formalismo, sendo mais flexíveis em relação à linguagem logico-matemática. Tanto a prova quanto à argumentação tem por finalidade o convencimento de que se está na direção da verdade. Podem partir de objetos pertencentes ao mundo real, tais como desenhos, gestos ou esboços. (SALES; PAIS, 2009).

Esses pontos de vista, em termos gerais, são correntes no meio acadêmico e escolar, embora possamos identificar no campo profissional, professores que não consideram o valor formativo desses processos como explicação e argumentação, possivelmente por possuírem compreensão confusa em relação aos conceitos.

Em função do objetivo deste texto, não aprofundaremos a discussão a respeito dos conceitos expostos acima. O fizemos em termos gerais para que pudéssemos ter parâmetros a respeito de tais conceitos no sentido de diferenciá-los de outros usos das palavras prova, demonstração e argumentação, comumente usadas na prática profissional do matemático e do professor de matemática. Assim, focalizaremos a discussão no pensamento de Wittgenstein a respeito da natureza das proposições matemáticas e das provas matemáticas, para em seguida discutirmos o uso dos gestos ostensivos como auxiliares no processo de prova em situações de ensino.

\footnotetext{
${ }^{1}$ Teoremas são proposições demonstráveis logicamente partindo de axiomas ou de outros teoremas já demonstrados mediante regras de inferência aceitas no campo científico. No contexto escolar, os teoremas têm valor de verdade por serem tratados à semelhança do campo científico, tanto em relação ao conceito, quando no ritual de demonstração.
} 
Wittgenstein $^{2}$ na fase madura de sua filosofia concebe a matemática como normativa e por esse fato, as provas matemáticas não poderiam ser pensadas de um ponto de vista de justificação externa ao que é provado. Por outras palavras, as argumentações da prova matemática não são de caráter empírico, mas estritamente gramaticais $^{3}$, o que garantiria que as proposições da matemática tenham sentido mesmo desvinculadas de usos externos à matemática (WITTGENSTEIN, 1967; WITTGENSTEIN, 2014).

Para o filósofo somente uma prova fornece a um enunciado matemático um sentido ou um conteúdo (FRASCOLLA, 2004), o que de certa forma evidencia a importância da explicação da prova e seu valor formativo em situação de ensino, pois, em consonância com o pensamento wittgensteiniano, descrever uma prova matemática em detalhes é já possuir a prova (JOURDAN, 2009). Por isso que a aprendizagem do sentido de uma fórmula matemática se caracteriza por uma aproximação gramatical do problema de aplicação, ou por outras palavras por uma explicação do sentido dos signos que compõem tal fórmula (SOUTIF, 2011).

Wittgenstein defende uma concepção de autonomia dos enunciados matemáticos que não se separe jamais de uma rede de relações conceituais do conteúdo, mesmo no caso de cálculos formais, como os cálculos aritméticos e geométricos. Sendo assim, uma proposição matemática não poderia jamais ser testada por via de um experimento (WITTGENSTEIN, 2014). Seria sim um constructo pelo qual a experiência pode ser testada e julgada, uma vez que os teoremas matemáticos exprimem regras gramaticais ou critérios para fazer asserções (BOUVERESSE, 1988; FRASCOLLA, 2004).

Nesse sentido, conjecturamos que o pensamento de Wittgenstein sobre as provas matemáticas o afastaria de pressupostos que sugerem que as provas de enunciados matemáticos em situações de ensino devam partir de situações do cotidiano, que leva os alunos a construir a prova matemática sem necessariamente fazer uso de argumentos logico-matemáticos, ou que utilize uma aplicação empírica como preparação para a compressão de uma proposição abstrata (WITTGENSTEIN, 2014).

\footnotetext{
${ }^{2}$ A filosofia de Wittgenstein é geralmente dividida em duas fases, a primeira refere-se às formulações presentes no Tractatus logico-philosophicus e a segunda refere-se aos seus escritos após 1933, época em que o filósofo tem como principal obra as Investigações filosóficas. Alguns comentadores ainda consideram um período intermediário do pensamento de Wittgenstein que engloba os escritos formulados entres as suas duas fases filosóficas.

${ }^{3} \mathrm{O}$ conceito de gramática para Wittgenstein se refere aos usos efetivos e aos usos possíveis das palavras, que se constroem em circunstâncias dialógicas (MORENO, 2005). No caso das proposições matemáticas, os usos das palavras são construídos no contexto social dos matemáticos e compartilhados pelos educadores matemáticos.
} 
Segundo o filósofo, devemos cuidar-nos de pensar que uma expressão como 4 maçãs +4 maçãs $=8$ maçãs é a equação concreta ou uma aplicação especial, da proposição abstrata $4+4=8$. Como exemplo de uma relação binária, costuma se dá algo parecido à relação pai e filho. Mas o que significa ter este exemplo para o tratamento lógico ulterior das relações binárias? Temos que imaginar "a é o pai de b" no lugar de qualquer "a R b"? Se este não é o caso, é este exemplo ou qualquer outro proveniente do mundo empírico essencial? Não joga este exemplo o mesmo papel que tem na aritmética o intento de explicar a alguém $3 \times 6=18$ usando 3 fileiras com 6 maçãs cada uma? (idem).

Wittgenstein entende que a matemática é essencialmente normativa, no sentido de que suas regras gramaticais têm sentido no interior do universo matemático independente de usos externos - a aritmética é sua própria aplicação, o cálculo é sua própria aplicação - mesmo assim, reconhece que a matemática pode ser utilizada como descritiva de alguma situação empírica, no entanto, em situações de ensino, a correlação entre os dois aspectos (normativo e descritivo) pode ir na direção de uma analogia que conduz a erros. (WITTGENSTEIN, 2014).

\section{A ostensão na filosofia de Wittgenstein}

A antiga doutrina de Wittgenstein, referente ao Tractatus Logico-Philosophicus, acolhe a ideia de que os elos básicos entre linguagem e mundo se constituem como simples relações biunívocas de denominação mediadas pelas formas lógicas (WITTGENSTEIN, 2001). Sendo assim, o meio característico de atribuição de significado é mostrar cada objeto e ao mesmo tempo pronunciar uma palavra lhe atribuindo um nome. Posteriormente, na fase madura, esse processo é denominado pelo filósofo de ensino ostensivo ou explicação ostensiva das palavras (WITTGENSTEIN, 1999).

Mesmo depois de afirmar que as concepções sobre a relação de denominação presentes no Tractatus não eram suficientes para dar conta de tudo o que podemos chamar de linguagem, o filósofo não excluiu a ostensão de sua nova maneira de pensar, pois apesar de perceber que as relações bipolares de denominação são insuficientes para a significação de algumas palavras, ele acredita que os gestos ostensivos podem evidenciar ao aprendiz a regra de uso da palavra a ser definida (WITTGENSTEIN, 1958). 
No primeiro capítulo do livro azul, Wittgenstein (1958) parece procurar um lugar em sua filosofia para alocar a definição ostensiva. Ele afirma que a definição ostensiva explica o sentido da palavra, porém a restringe, dizendo que não existem definições ostensivas para muitas palavras na nossa linguagem, tais como "um", "número", "não", etc. O que ele quer evidenciar é que a definição ostensiva efetiva a compreensão do sentido da palavra, mas não predicam algo do definido. Ela pode ser interpretada, ou dizendo melhor, pode ter múltiplas interpretações.

Mas o que leva a essas múltiplas interpretações, sobretudo quando o objeto apontado pertence ao universo da matemática? O motivo mais evidente nos parece que se refere ao fato de que na nova maneira de pensar do filósofo, já no período intermediário, as ligações linguagem-mundo já não são vistas como relações biunívocas, portanto, em casos em que não ocorre a correspondência dessa natureza entre nome e denominado, como nos casos de substantivos tais como "mesa", "cadeira", etc., a definição ostensiva pode ser mal compreendida (WITTGENSTEIN, 1999). Por exemplo: quando o professor aponta para o símbolo " $\square$ " no interior de um retângulo e diz "isto é um ângulo reto", é preciso que o ouvinte conheça a condição lógica da entidade definida para saber identificar o aspecto apontado pelo professor.

Já na fase madura, nas Investigações filosóficas, Wittgenstein apresenta exemplos em que recomenda a elucidação ostensiva dessas condições lógicas como recurso para evitar interpretações errôneas a fim de efetivar a compreensão da palavra e consequentemente fazer com que a definição ostensiva cumpra o seu papel de elucidar o uso $^{4}$ - a significação - da palavra (WITTGENSTEIN, 1999).

Nessa obra, no conjunto de aforismos que vai do sexto ao trigésimo primeiro, o filósofo fala em ensino ostensivo das palavras e definição ostensiva e apresenta variados exemplos para que o leitor possa compreender a função de cada conceito na sua maneira de pensar, bem como a relação entre os dois conceitos, no ensino e na aprendizagem da linguagem. É o que iremos discutir na seção seguinte.

\section{Ensino ostensivo e definição ostensiva: semelhanças e diferenças}

A atribuição de nomes é um tema presente na primeira parte das Investigações Filosóficas, em que Wittgenstein discute dois aspectos do processo de organização

\footnotetext{
${ }^{4}$ Para Wittgenstein, conceito de uso diz respeito às finalidades e ao funcionamento da palavra em situações de sua aplicação (MORENO, 2005, p.153). Neste texto, sempre que o termo aparecer para se referir a uso, no sentido de uso das palavras, fica implícito que a utilização é nos termos de Wittgenstein.
} 
linguística da experiência através da atribuição de nomes, a saber, os pontos de contato entre linguagem e realidade e a natureza e constituição da significação proposicional, temas já trabalhados no Tractatus, e que são retrabalhados nas Investigações, sob a forma de ensino ostensivo das palavras e definição ostensiva (MORENO, 2012).

De acordo com Hinttika (2004), pode-se identificar uma quantidade significativa de referências à ostensão nas obras do período intermediário The blue book, Wittgenstein's lectures, Cambridge 1932-1935 e Philosophical grammar, bem como uma ênfase renovada em seu papel de ligação linguagem-mundo em relação ao Tractatus.

Já na fase madura, o ensino ostensivo é apresentado como uma técnica capaz de adestrar o comportamento para fazer associações de imagens mentais a objetos e para facilitar a compreensão das palavras por meio de lições. Segundo o filósofo, as lições em geral se enquadram no seguinte processo: o que aprende denomina os objetos. Fala a palavra quando o professor aponta para o objeto (WITTGENSTEIN, 1999).

Apesar de Wittgenstein afirmar que o ensino ostensivo estabelece uma ligação associativa entre a palavra e a coisa, não devemos concluir que ele está limitando o ensino ostensivo à função referencial que lhe é tradicionalmente atribuída. Ele enfatiza que esse processo é importante como treinamento por ser recorrente entre as pessoas, mas que não é a única maneira de se ensinar as palavras. O que o filósofo destaca como importante nesta técnica é o fato de que ela efetiva a compreensão da palavra (WITTGENSTEIN, 1999).

Já no segundo aspecto da nomeação, referente à natureza e à constituição da significação proposicional, ou por outras palavras à pergunta pelo sentido ou à definição ostensiva do nome, o aprendiz deve dominar alguns dos diversos usos que podem ser feitos com o nome, para saber qual o aspecto da palavra que está sendo apontado - por exemplo, a forma e não a cor ou outro aspecto do objeto (WITTGENSTEIN, 1999).

Por outras palavras, o aprendiz já seria capaz de refletir criticamente sobre a denominação, por isso trata-se de definição e não de ensino ostensivo. Porém, em ambos os casos se requer uma familiaridade prévia com o uso das palavras, por meio de lições, chamada pelo filósofo de lições de linguagem (WITTGENSTEIN, 1999). Isso conduz o aprendiz a interpretar as relações de sentido nas novas lições que lhe forem impostas. Por exemplo, a palavra "água" usada como denominação tem sentido imediato para o aprendiz, mas o que dizer a respeito do sentido da exclamação "água!"? 
Segundo Moreno (2014), o aprendiz deve ser capaz de distinguir entre a aplicação do nome como etiqueta, como exclamação, como pedido ou apelo ou como evocação de imagens mentais, dentre outras.

A definição ostensiva só poderá esclarecer o uso do nome quando já é claro para o aprendiz o papel que o nome desempenha na linguagem. Por exemplo, na definição ostensiva da palavra dois: "Este número chama-se dois", a palavra número nesta definição indica o local da linguagem em que o professor está colocando a palavra, mas o aprendiz já deve conhecer o significado da palavra número dentro desse contexto, por meio do ensino ostensivo (WITTGENSTEIN, 1999).

\section{Gestos ostensivos: auxiliares na prova matemática}

De acordo com Moreno (2005) podemos criar enunciados hipotéticos, descritivos e explicativos a partir da definição ostensiva de conceitos que nos permite fazer recortes da experiência. A partir da definição ostensiva de uma cor, ou de uma gramática de cores, indicar quais as combinações possíveis e impossíveis entre as cores, para dar resposta à questão o que é uma determinada cor. Ou o que é uma sensação ou emoção a partir da definição ostensiva de sensações ou emoções, ou de uma gramática de estados mentais, e indicar as combinações possíveis e impossíveis entre eles.

Segundo o autor, esse é o processo usual de levantamento de hipóteses sobre as cores do universo de cores e sobre os estados mentais do universo psíquico. Os modelos científicos também se utilizam desse recurso para explicar regiões da experiência - o sentido dessas regiões já é conhecido de maneira imprecisa e vaga, por meio de vagas e imprecisas definições ostensivas e de gramáticas conceituais que refletem e cristalizam usos de palavras em situações pragmáticas de sua aplicação.

O mesmo vale para as entidades abstratas da matemática e da lógica, assim como para diferentes formas de raciocínio nelas desenvolvidas - os números, o infinito, as relações de identidade, implicação, equivalência, as formas lógicas, as provas e as demonstrações, a verdade e a contradição etc. (MORENO, 2005).

Em relação às provas matemáticas, Wittgenstein (2014) afirma que o conceito de prova de uso corrente entre os matemáticos é circunscrito tão somente por provas particulares ou por séries de provas análogas a séries numéricas. Segundo o filósofo com a definição da palavra prova ocorre o mesmo que com a definição da palavra número: pode-se definir a expressão número cardinal mostrando exemplos de números cardinais, e até fazer uso dos signos $1,2,3, \ldots$, ad inf. em lugar dessa expressão. 
De outra maneira, a palavra número pode ser definida mostrando-se diferentes tipos de número, mas com isso não estamos circunscrevendo o conceito de número tão definidamente como antes foi com o de número cardinal, a não ser que se queira dizer que unicamente o que chamamos de número constitui o conceito de número. Mas nesse caso, de nenhuma construção nova se pode dizer que é a construção de uma espécie de números.

Aprendemos a verificabilidade de uma equação em sua prova, da mesma maneira que é na figura, onde aprendemos a verificabilidade de uma proposição geométrica. Ver a prova verá então o que aqui é demonstrado, o que se chama a proposição demonstrada (WITTGENSTEIN, 2014), ou seja, descrever uma prova é provar.

O mostrar nesse processo se refere ao uso dos gestos ostensivos, que de acordo com a filosofia Wittgensteiniana, vem acompanhado do falar na lição de linguagem, que no caso aqui discutido, se trata de provar um enunciado matemático aos alunos.

Como ilustração, apresentaremos um exemplo em que os gestos ostensivos podem ser utilizados como auxiliares no processo de prova da equação abaixo em que $x$ e $y$ pertencem aos números reais e $n$ pertence aos números naturais:

$$
(x+y)^{n}=\left(\begin{array}{c}
n \\
0
\end{array}\right) x^{n}+\left(\begin{array}{c}
n \\
1
\end{array}\right) x^{n-1} y+\left(\begin{array}{c}
n \\
2
\end{array}\right) x^{n-2} y^{2}+\cdots+\left(\begin{array}{l}
n \\
k
\end{array}\right) x^{n-k} y^{k}+\cdots+\left(\begin{array}{l}
n \\
n
\end{array}\right) y^{n}
$$

A verificabilidade dessa igualdade é mostrada pelos professores como prova de que o desenvolvimento de qualquer binômio desse tipo pode ser obtido dessa maneira. Para fins pedagógicos, a descrição da verificabilidade da igualdade tem duplo objetivo: provar a proposição e apresentar uma técnica para o desenvolvimento de qualquer binômio particular.

Em geral, pode-se observar a seguinte estratégia para empreender a prova da verificabilidade da igualdade ${ }^{5}$ : o professor procura trazer à memória dos alunos conceitos e técnicas, tais como potência, produtos notáveis, e temas da análise combinatória, estudados anteriormente por eles, para auxiliar na compreensão da técnica de prova comumente usada, sobretudo na educação básica. A prova é empreendida por meio da descrição de casos particulares, na direção do caso geral.

\footnotetext{
${ }^{5}$ Esse procedimento pode ser encontrado em livros didáticos do ensino médio e em alguns do ensino superior.
} 
A estratégia consiste em conduzir o aluno a fazer analogias a partir do desenvolvimento de potências em que a base é o binômio $(x+y)$ e o expoente assume os valores do parâmetro para $n$ por meio de uma sequência de exemplos:

Para $n=2$, obtém-se o seguinte desenvolvimento: $(x+y)^{2}=x^{2}+2 x y+y^{2}$.

Sendo $n=3$, obtém-se o desenvolvimento: $(x+y)^{3}=x^{3}+3 x^{2} y+3 x y^{2}+y^{3}$.

Em seguida o professor deve escrever os coeficientes e expoente subtendidos e chamar a atenção do aluno para cada termo do desenvolvimento. Os coeficientes e expoentes devem ser mostrados, inclusive os que estavam subtendidos podem ser evidenciados por meio do gesto ostensivo:

$$
\begin{aligned}
& (x+y)^{2}=x^{2}+2 x y+y^{2}=1 x^{2} y^{0}+2 x^{1} y^{1}+1 x^{0} y^{2} \\
& (x+y)^{3}=x^{3}+3 x^{2} y+3 x y^{2}+y^{3}=1 x^{3} y^{0}+3 x^{2} y^{1}+3 x^{1} y^{2}+1 x^{0} y^{3}
\end{aligned}
$$

Após evidenciar os coeficientes e expoentes subtendidos, o professor deve elucidar por meio do gesto ostensivo a relação de equivalência entre os coeficientes do polinômio obtido e os números binomiais que são definidos de acordo com o expoente $n$, bem como o comportamento dos expoentes dos termos $x$ e $y$, em um processo de elucidação ostensiva, falando enquanto mostra os termos equivalentes (se for necessário o professor pode usar um espaço no quadro para mostrar essas equivalências).

$$
\begin{aligned}
& (x+y)^{2}=x^{2}+2 x y+y^{2}=1 x^{2} y^{0}+2 x^{1} y^{1}+1 x^{0} y^{2}=\left(\begin{array}{l}
2 \\
0
\end{array}\right) x^{2} y^{0}+\left(\begin{array}{l}
2 \\
1
\end{array}\right) x^{1} y^{1}+\left(\begin{array}{l}
2 \\
2
\end{array}\right) x^{0} y^{2} \\
& (x+y)^{3}=1 x^{3} y^{0}+3 x^{2} y^{1}+3 x^{1} y^{2}+1 x^{0} y^{3}=\left(\begin{array}{l}
3 \\
0
\end{array}\right) x^{3} y^{0}+\left(\begin{array}{l}
3 \\
1
\end{array}\right) x^{2} y^{1}+\left(\begin{array}{l}
3 \\
2
\end{array}\right) x^{1} y^{2}+\left(\begin{array}{l}
3 \\
3
\end{array}\right) x^{0} y^{3}
\end{aligned}
$$

A partir deste processo espera-se que o aluno seja capaz de desenvolver e apresentar a forma polinomial de outros binômios que se enquadram nos parâmetros apresentados. Já o professor deverá se valer dessa capacidade que os alunos possam ter desenvolvido para empreender o desenvolvimento do binômio genérico $(x+y)^{n}$ e ter sua prova concretizada.

Mas vejamos: o aluno só poderia compreender o sentido da prova, o uso do termo binômio de Newton e as técnicas apresentadas somente desta maneira? Certamente não. Porém, se a prova for empreendida assim, algumas palavras devem ser elucidadas previamente para que o aluno tenha condições para compreender o processo de prova e de desenvolvimento da técnica apresentada.

Em conformidade com Wittgenstein, as palavras potência e binômio deveriam ser elucidadas por meio do ensino ostensivo, antes que o professor pronuncie a frase “isto se chama 'binômio de Newton"”, apontando para a expressão matemática $(x+y)^{n}$. 
Pode ser necessário elucidar ostensivamente outros elementos presentes na expressão e em suas condicionantes tais como universo, coeficiente, termo, número binomial, etc., pois a compreensão do significado/uso dessas palavras pode evitar que alguém compreenda de modo diverso da lógica da matemática. Agindo assim, o professor poderá evitar mal-entendidos. Segundo o filósofo, o professor deve construir uma cadeia de elucidações, em que sempre se pode construir mais uma elucidação, caso seja necessário, indicando o uso da palavra. (WITTGENSTEIN, 1999).

Neste caso, a definição ostensiva tanto elucida o uso do termo binômio de Newton (relacionado à técnica do desenvolvimento) quanto apresenta as regras do jogo de linguagem da prova, considerando que em tal jogo "um parceiro enuncia as palavras, o outro age de acordo com elas" constituindo assim uma forma de vida (WITTGENSTEIN, 1999, p. 18). Wittgenstein diria que a definição ostensiva só esclarece o uso de uma palavra quando o aprendiz já domina diversos jogos com a respectiva palavra, ou ainda que é necessário que o aprendiz domine técnicas introdutórias para que possa fazer relação de familiaridade entre os jogos de linguagem e os usos das palavras (WITTGENSTEIN, 1999).

\section{Considerações finais}

Neste artigo, discorremos sobre o uso dos gestos ostensivos como auxiliares no processo de explicação da prova de enunciados matemáticos em situações de ensino e de aprendizagem. Os gestos ostensivos na filosofia do segundo Wittgenstein podem servir tanto ao processo de nomeação puramente referencial, na forma de ensino ostensivo, quanto na definição ostensiva do nome, ou ainda à pergunta pelo sentido.

Como foi evidenciado, os gestos ostensivos podem auxiliar tanto o processo de demonstração quanto o de compreensão dos teoremas e enunciados matemáticos em geral, pois podem elucidar o uso das palavras e consequentemente lhes conferir sentido e conteúdo, uma vez que uma prova não é somente uma sequência de etapas que nos obriga a reconhecer a verdade de uma frase tendo um sentido independente dela.

A reflexão a respeito das observações filosóficas de Wittgenstein nos permitiu inferir que o esforço do filósofo na fase intermediária para delimitar os limites da ostensão é decorrente, em parte, do fato do gesto ostensivo ser um recurso linguístico muito presente tanto no processo de nomeação, quanto em jogos de linguagem mais 
complexos. Como afirma o experiente Wittgenstein nas Investigações filosóficas "isso ocorre entre os homens" (WITTGENSTEIN, 1999).

Em situações de ensino, nos processos de explicação, de argumentação e de prova é evidente a necessidade de utilização dos gestos ostensivos como recurso auxiliar no ensino do uso das palavras e na produção de sentido. No caso da prova, os gestos ostensivos poderão servir para trazer à memória do aprendiz técnicas que o permitirão interpretar o jogo de linguagem da prova e ser capaz de compreender novas técnicas e aplicá-las adequadamente.

No exemplo apresentado, a explicação ostensiva deve colocar em cena a técnica da potência, o desenvolvimento dos binômios $(x+y)^{2}$ e $(x+y)^{3}$ pela técnica dos produtos notáveis, a determinação do valor de números binomiais, além da correspondência referencial dos números binomiais aos coeficientes dos polinômios pelo ensino ostensivo.

A elucidação destas técnicas e dos conceitos relacionados tem um importante valor formativo que vai além da resposta à pergunta que movimenta a prova: "por que é verdadeiro?". O ir além da prova se evidencia no fato de que na prova seguimos regras. Como evidenciado nesta discussão, a apresentação de gramáticas conceituais por meio de definições ostensivas das entidades abstratas da matemática no processo de prova esclarecem os usos que fazemos das palavras e, uma vez sistematizados como regras, esclarecem a significação dos conceitos e dos enunciados matemáticos.

\section{Referências}

ALMOULOUD, S. A.; FUSCO, C. A. S. Provas e Demonstrações em Matemática: uma questão problemática nas práticas docentes no ensino básico. In: ENCONTRO NACIONAL DE EDUCAÇÃO MATEMÁTICA, 10., 2010, Salvador-BA. Anais... 2010, CD-ROM.

ALMOUlOUD, S. A.; SILVA, M. F.; FUSCO, C. A. S. Provar e Demonstrar: um espinho nos processos de ensino e aprendizagem da matemática. Revista Paranaense de Educação Matemática, v.1, n.1, p. 22-41, 2012.

BALACHEFF, N. Processus de Preuve et Situations de Validation. Educational Studies in Mathematics, v. 18, n. 2, p. 147-176, 1987.

BOUVERESSE, J. Le pays des possibles: Wittgenstein, les mathématiques et le monde réel. Paris: Les Édidions Minuit, 1988.

BRASIL. Parâmetros curriculares Nacionais Ensino Médio: Ciências da Natureza, Matemática e suas Tecnologias. Secretaria de Educação Média e Tecnológica Brasília: MEC/SEB, 2000. 
FRASCOLLA, P. Wittgenstein sur la Preuve mathematique. In: FLOYD, J. et al. Wittgenstein et les mathématiques. Paris: T. E. R., 2004. p. 43-60.

HINTTIKA, J. Da ostensão aos jogos de linguagem. In: HINTIKKA, M; HINTIKKA, J. Uma investigação sobre Wittgenstein. São Paulo, Papirus, 1994.

JOURDAN, C. Provas matemáticas em Wittgenstein. Revista Filosofia Aurora, v. 21, n. 29, p. 297-312, 2009.

LACERDA, A. G. Interpretação e comunicação das regras matemáticas na resolução de problemas de divisão por alunos da $5^{a}$ série do ensino fundamental. Dissertação de mestrado em Educação em Ciências e Matemáticas - Universidade Federal do Pará, Belém, 2010.

MERLI, R. F. Modelos clássico e Fuzzy na Educação Matemática: um olhar sobre o uso da linguagem. Dissertação de mestrado em Ensino de Ciências e Educação Matemática - Universidade Estadual de Londrina, Londrina, 2012.

MORENO, A. R. Introdução a uma epistemologia do uso. Caderno $C R H$, v. 25, n. 2, p. 73-95, 2012.

MORENO, A. R. Introdução a uma pragmática filosófica: de uma concepção de filosofia como atividade terapêutica a uma filosofia da linguagem. Campinas-SP: Editora da Unicamp, 2005.

OLIVEIRA, M. S. Interpretação e comunicação em ambientes de aprendizagem gerados pelo processo de modelagem matemática. Dissertação de mestrado em Educação em Ciências e Matemáticas - Universidade Federal do Pará, Belém, 2010.

SALES, A.; PAIS, L. C. A Argumentação no Estudo da Geometria: Uma Experiência com Acadêmicos de Licenciatura em Matemática. In: ENCONTRO BRASILEIRO DE ESTUDANTES DE PÓS-GRADUAÇÃO EM EDUCAÇÃO MATEMÁTICA, 13., 2009, Goiânia-GO. Anais... Goiânia, 2009. CD-ROM.

SOUZA, H. C. T. Uma análise dos esquemas do processo de modelagem. Dissertação de mestrado em Educação em Ciências e Matemática - Universidade Federal do Paraná, Curitiba, 2013.

SOUTIF, L. Applications des calculs mathématiques et explication ostensive: deux conceptions de l'autonomie; Wittgenstein sur l'application. In: Wittgenstein et le problème de l'espace visuel. Paris: VRIN, 2011, p. 206-220.

SILVA, P. V. O aprendizado de regras matemáticas: uma pesquisa de inspiração wittgensteiniana com crianças da $4^{a}$ série no estudo da divisão. Dissertação de mestrado em Educação em Ciências e Matemáticas - Universidade Federal do Pará, Belém, 2011. 
TORTOLA, E. Os usos da linguagem em atividades de modelagem matemática nos anos iniciais do ensino fundamental. Dissertação de mestrado em Ensino de Ciências e Educação Matemática - Universidade Estadual de Londrina, Londrina, 2012.

VILELA, D, S. Elementos para uma compreensão das matemáticas como práticas sociais. In: MIORIM, M. A.; VILELA, D. S. História, filosofia e educação matemática: práticas de pesquisa. Campinas-SP: Editora Alinea, 2009. p. 89-126.

WITTGENSTEIN, L. The Blue and Brown books. Oxford: Blackwell, 1958

WITTGENSTEIN, L. Remarks on foundations of mathematics. Edited by G.H.von Wright, R. Rhees and G. E. M. Anscombe. Translated by G. E. M. Anscombe. Oxford: Blackwell, 1967.

WITTGENSTEIN, L. Investigações Filosóficas. Trad. José Carlos Bruni. São Paulo: Nova Cultural, 1999.

WITTGENSTEIN, L. Tractatus Lógico-Philosophicus. São Paulo: EDUSP, 2001.

WITTGENSTEIN, L. Escrito a máquina [The Big Typescript]. Madrid: Editorial Trotta, 2014.

MARCELO DE SOUSA OLIVEIRA. Doutorando em Educação em Ciências e Matemáticas do Programa de Pós-Graduação do Instituto de Educação Matemática e Científica, da Universidade Federal do Pará. Professor Assistente da Faculdade de Matemática do Instituto de Ciências Exatas da Universidade Federal do Sul e Sudeste do Pará. Integrante do Grupo de Estudo e Pesquisa em Linguagem Matemática (GELIM/UFPA) e do Grupo de Estudo e Pesquisa em Educação Matemática (GEPEM/UNIFESSPA).

MARISA ROSÂNI ABREU DA SILVEIRA. Doutora em Educação pela Universidade Federal do Rio Grande do Sul, com estágio na Universidade de Paris. Professora da Faculdade de Educação Matemática e Científica, no curso de Licenciatura Integrada em Educação em Ciências, Matemática e Linguagens, do Programa de PósGraduação em Educação em Ciências e Matemáticas da Universidade Federal do Pará e vice coordenadora do Polo Acadêmico REAMEC/UFPA. Líder do Grupo de Estudo e Pesquisa em Linguagem Matemática (GELIM/UFPA).

Recebido: 19 de dezembro de 2015

Revisado: 08 de maio de 2016

Aceito: 02 de junho de 2016 